\title{
PERFORMANCE EVALUATION OF A GIGABIT DSL MODEM USING SUPER ORTHOGONAL COMPLETE COMPLEMENTARY CODES UNDER PRACTICAL CROSSTALK CONDITIONS
}

\author{
J.H. van Wyk* and L.P. Linde ${ }^{\dagger}$ \\ * Department of Electrical, Electronic \& Computer Engineering, University of Pretoria, South Africa, \\ 0002.E-mail: jhvanwyk@up.ac.za \\ $\dagger$ Department of Electrical, Electronic \& Computer Engineering, University of Pretoria, South Africa, \\ 0002.E-mail: lplinde@ieee.org
}

\begin{abstract}
This paper describes the unique performance evaluation results of a Gigabit Digital Subscriber Line modem that provides Multi-user-interference-free communication, by incorporating Super Orthogonal Complete Complementary spreading into the existing xDSL modem architecture. The GDSL modem was tested under practical crosstalk conditions, using the Network Interface, Power, and Protection (NIPP) Committee MIMO Crosstalk Model. It was found that the GDSL modem provides acceptable bit error rate performance for a fully loaded system, even for very short twisted pairs. A unique observation from these performance evaluation tests is than the non-linear behaviour of NEXT and FEXT creates an orthogonality distortion effect on the correlation properties of the SOCC code family.
\end{abstract}

Keywords: Gigabit Digital Subscriber Line (GDSL), Super Complete Complementary (SOCC), MUI-free, Performance evaluation, MIMO crosstalk, FEXT, NEXT, crosstalk

\section{INTRODUCTION}

In this paper we present the unique performance evaluation results of a Gigabit Digital Suscriber Line (GDSL) modem that was developed and described in [1], where a "spreading block" (SB) is added to the existing DSL architecture, using Super Orthogonal Complete Complementary (SOCC) spreading codes to create a Multi-user Interference (MUI) free environment, even in the presence of severe FEXT crosstalk (very short loops).

In Section 2 the proposed system architecture is briefly described from [1]. In Section 3 the channel modeling, NEXT and FEXT crosstalk environment and the NIPP MIMO Crosstalk model is provided. In Section 4 the performance evaluation results is provided and discussed. Finally a conclusion of this paper is provided in Section 5.

\section{PROPOSED SYSTEM ARCHITECTURE}

The proposed GDSL system architecture was described in a previous paper [1], but is repeated here for completeness. Figure 1 shows the GDSL transmitter [2], based on Discrete Multi-Tone (DMT), with the proposed SOCC spreader highlighted. Figure 2 shows the corresponding GDSL receiver. With a subchannel spacing of $51.75 \mathrm{kHz}$ $(12 \times 4.3125 \mathrm{kHz})$ and using a 4096 FFT structure, a total system bandwidth of $211.968 \mathrm{MHz}$ is required. This bandwidth is suggested for future G.fast systems [3]. During modem initialization the channel attenuation and background noise is determined. For our simulations,

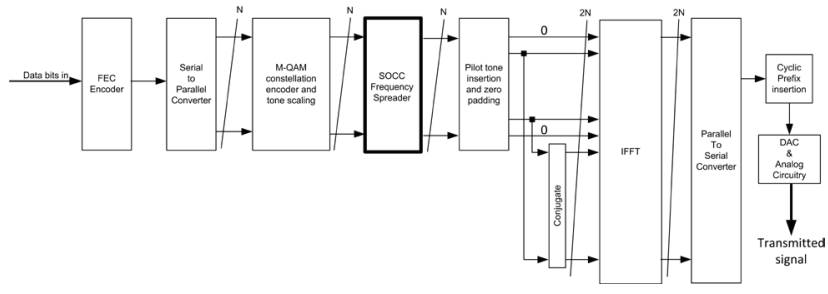

Figure 1: GDSL Transmitter with proposed SOCC Spreader (highlighted) [Taken from [1]]

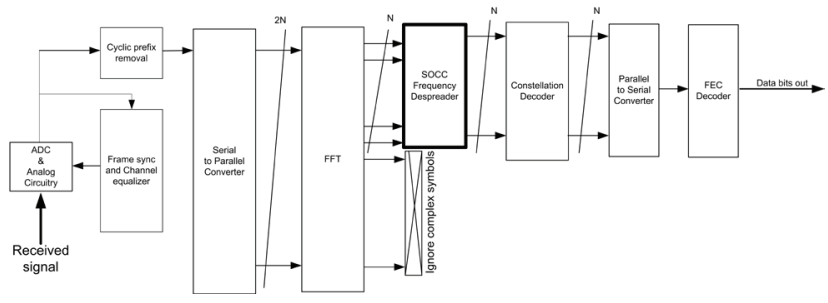

Figure 2: GDSL Receiver with proposed SOCC Despreader (highlighted) [Taken from [1]]

a theoretical approach, based on two-port networks will be used, as discussed in Section 3. Figure 3 shows the Power spectral densities of the pilot tones (in this example $-60 \mathrm{dBm} / \mathrm{Hz}$ ), the channel attenuation (shown in $\mathrm{dB}$ to compare profile with that of received tones), the received tones, the background noise, $99 \%$ worst case NEXT noise level and 99\% worst case FEXT noise levels for different coupling lengths $d$, for $200 \mathrm{~m}$ of $0.5 \mathrm{~mm}$ twisted pair. 


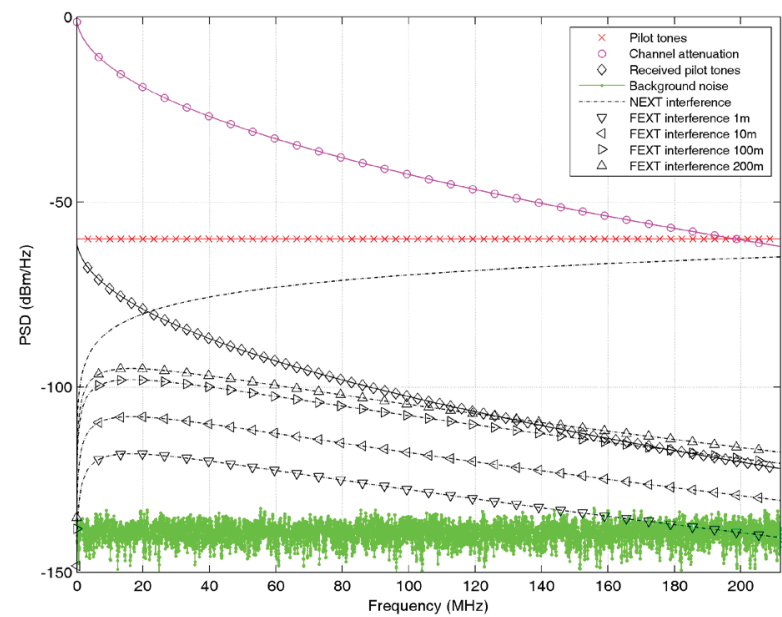

Figure 3: Power spectral density of pilot tones, channel attenuation (in $\mathrm{dB}$ ), received tones, background noise, $99 \%$ worst case NEXT noise level and 99\% worst case FEXT noise levels for different coupling lengths.

\section{CHANNEL AND CROSSTALK ENVIRONMENT}

\subsection{Channel modeling}

Local loops usually consist of several sections of cable with different lengths and wire gauges, with or without bridged taps, and terminated with resistive impedance. Two-port networks, and specifically ABCD matrixes can be used to represent each segment or section of a line. By multiplying the ABCD matrixes of each segment, an ABCD matrix is obtained which represents the complete line [2]. For a $200 \mathrm{~m} 0.5 \mathrm{~mm}$ twisted-pair, the obtained channel attenuation is shown in Figure 4. A practical xDSL modem will obtain a SNR profile of the channel. This is referred to as the measured SNR ( $\left.S N R_{\text {meas }}\right)$. To allow for performance variations and a target uncoded BER of $10^{-7}$, a SNR performance margin of $\Gamma=10 \mathrm{~dB}$ is added to $S N R_{\text {meas }}$ to obtain $S N R_{\text {used }}$ [2], which is used by the modem. A practical example, with a VDSL 30a profile bypass policy, averaged over 518 FFT blocks (10 ms) is shown in Figure 4.

\subsection{NEXT and FEXT modeling}

The transfer function for a $99 \%$ worst-case NEXT channel can be expressed as [2]:

$$
\left|H_{N E X T, 99}(f)\right|^{2}=x_{n} \cdot f^{1.5}
$$

and the PSD as:

$$
P S D_{N E X T}=P S D_{D i s} \cdot\left|H_{N E X T, 99}(f)\right|^{2}
$$

where $x_{n}=8.814 \cdot 10^{-14}$ and $f$ is the frequency [Hz].

FEXT is dependent on the characteristics of the line. The original signal at the transmitter (of a disturber) will be

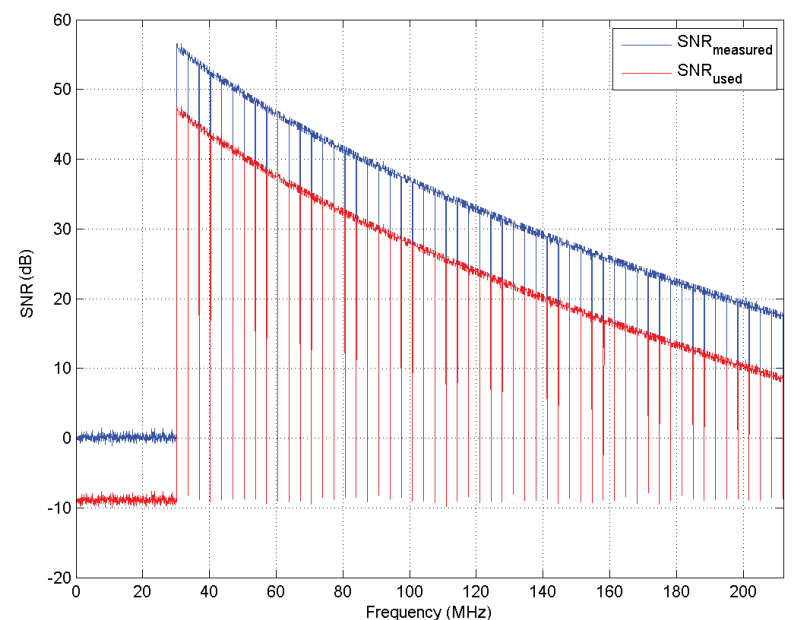

Figure 4: $S N R_{\text {meas }}$ and $S N R_{\text {used }}$ for a $200 \mathrm{~m} 0.5 \mathrm{~mm}$ twisted copper pair

attenuated due to the inherent propagation loss of the line. In a real network, FEXT is not just a function of the crosstalk in the cable, but also of the cable topology [2], i.e.

$$
\left|H_{F E X T, 99}(f)\right|^{2}=x_{n} \cdot l \cdot\left|H_{\text {ins }}(f)\right|^{2} \cdot f^{2}
$$

and the PSD as:

$$
P S D_{F E X T}=P S D_{D i s} \cdot\left|H_{F E X T, 99}(f)\right|^{2}
$$

where $x_{n}=2.625 \cdot 10^{-16}, l$ is the length of the disturbing line $[\mathrm{m}], H_{\text {ins }}(f)$ is the insertion loss for the line under consideration, $|\cdot|^{2}$ is the modulus-squared function, and $f$ is the frequency $[\mathrm{Hz}]$.

In Figure 5 a comparison is shown between NEXT, $\operatorname{FEXT}(l=0 \mathrm{~m}), \operatorname{FEXT}(l=100 \mathrm{~m})$ and $\operatorname{FEXT}(l=200 \mathrm{~m})$. It

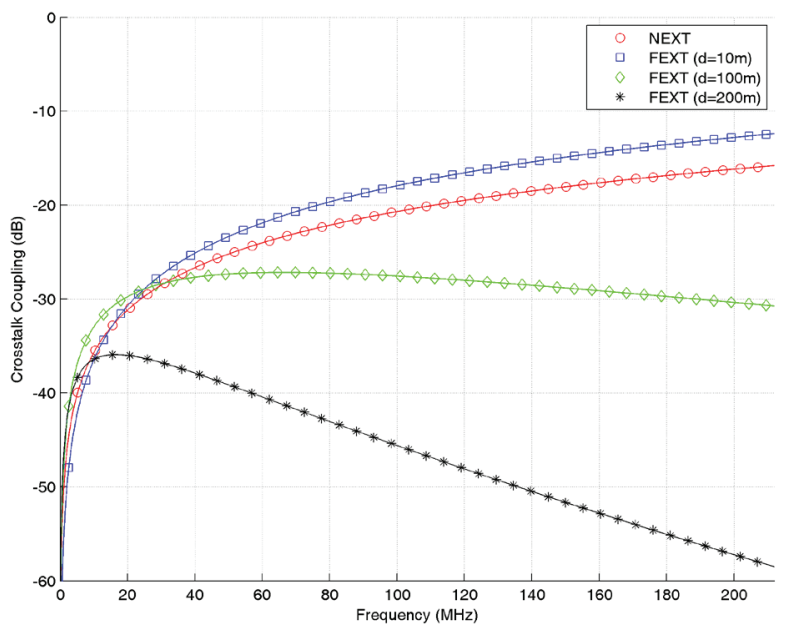

Figure 5: NEXT and FEXT Crosstalk coupling

should be noted that FEXT approaches NEXT as the line length decreases (with the coupling length equal to the 


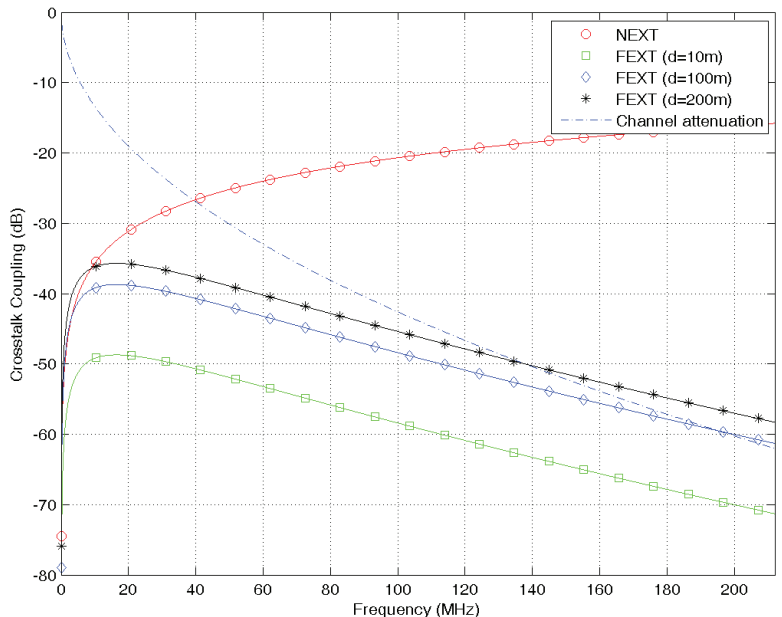

Figure 6: NEXT and FEXT Crosstalk coupling comparison for $200 \mathrm{~m} 0.5 \mathrm{~mm}$ twisted pair for different coupling lengths

line length). In Figure 6 a comparison is shown between NEXT, FEXT $(\mathrm{d}=10 \mathrm{~m})$, FEXT $(\mathrm{d}=100 \mathrm{~m})$ and FEXT $(\mathrm{d}=200 \mathrm{~m})$, for a $200 \mathrm{~m} 0.5 \mathrm{~mm}$ twisted-pair.

In ADSL systems, where the line length was still relatively long (the so called 'last-mile'), NEXT was eliminated by using a FDD approach, using different sub-channels for upstream and downstream transmission. One approach to control crosstalk was to control certain transmission parameters such as tone power levels. These "spectrum management" restrictions are conservatively designed so that $99 \%$ of all operational cases operate properly. FEXT had PSD levels close to or below the noise level due to large line attenuation, specifically at higher frequencies. With VDSL / VDSL2 the FEXT from other VDSL systems (often called 'self-FEXT') is the major performance constraining factor, especially as the loop length becomes shorter. As the line attenuation decreases for shorter loops, FEXT noise starts to approach the same levels as NEXT noise. NEXT is usually avoided by using a FDD approach (upstream and downstream bands in separate frequency bands) or a TDD (sending upstream packets and downstream packets after each other) approach.

FEXT remains a problem for the FDD approach - a solution was to apply dynamic spectral management (DSM) Level 1. DSM Level 2 performs spectrum balancing jointly across multiple lines to mitigate crosstalk, while DSM Level 3 applies Vectored DSL to effectively remove crosstalk. Vectored DSL makes use of pre-coding in downstream transmission and makes use of Multi-User Detection (MUD) interference cancellation in upstream transmission [4]. For the GDSL modem, SOCC spreading is used to mitigate the effect of crosstalk (MUI).

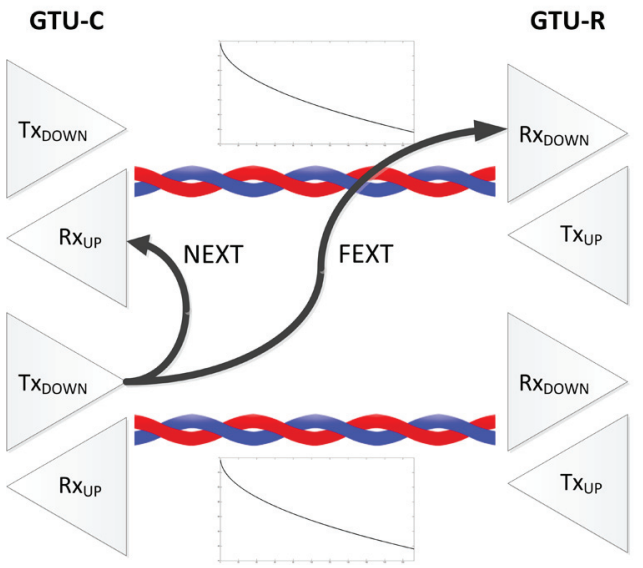

User $i$

Figure 7: Illustration of NEXT and FEXT interference

Consider Figure 7. In order to determine how SOCC spreading will perform under NEXT and FEXT conditions, the disturbing signal (including background noise, NEXT interferer and or FEXT interferer) should be considered. It is equivalent to determining the Interference noise. Consider the case of sending information downstream for User $i$. If $T x_{D O W N}$ has a power spectrum of $S_{D O W N}(f)$, the transmitter's received version at the downstream receiver $\left(R x_{D O W N}\right)$ will be $S_{D O W N}(f)$. $\left|H_{\text {ins }}(f)\right|^{2}$, basically an attenuated version of the transmitter's spectrum. The interfering received signal from User(s) $j$ will be a power sum of NEXT and FEXT terms [5], each respectively of the form $S_{U P}(f) . N E X T(f)$ and $S_{D O W N}(f) . F E X T(f)$. If $N_{o} / 2$ is the background noise, the Interference noise will be:

$$
\begin{aligned}
N_{\text {int }} & =\frac{N_{0}}{2}+\left(\frac{N_{j}}{N_{\text {dis }}}\right)^{0.6} S_{u p}^{j}(f) \cdot N E X T(f) \\
& +\left(\frac{N_{j}}{N_{\text {dis }}}\right)^{0.6} S_{\text {down }}^{j}(f) \cdot F \operatorname{EXT}(f)
\end{aligned}
$$

for all Users $j$, where $N_{j}$ is the number of similar disturbers from Users $j$ and $N_{d i s}$ is the total number of disturbers. During modem initialization the channel attenuation profile is determined and synchronized between the GTU-C and GTU-R. Since both receivers of User $i$ have knowledge of the channel, the 1-tap decision feedback equalizer (DFE) can remove the effect of insertion loss. This is equivalent to dividing the received signal by $\left|H_{\text {ins }}(f)\right|^{2}$. The equalized Interference noise then becomes:

$$
\begin{aligned}
N_{\text {int }} & =\frac{\frac{N_{0}}{2}}{\left|H_{\text {ins }}^{i}(f)\right|^{2}} \\
& +\left(\frac{N_{j}}{N_{\text {dis }}}\right)^{0.6} \frac{S_{u p}^{j}(f) \cdot N E X T(f)}{\left|H_{\text {ins }}^{i}(f)\right|^{2}} \\
& +\left(\frac{N_{j}}{N_{\text {dis }}}\right)^{0.6} \frac{S_{\text {down }}^{j}(f) \cdot F E X T(f)}{\left|H_{\text {ins }}^{i}(f)\right|^{2}}
\end{aligned}
$$

where $N_{j}$ is the number of similar disturbers from Users $j$ and $N_{d i s}$ is the total number of disturbers. 
From Figure 6, NEXT and FEXT (for short loops) provide the most detrimental (crosstalk) effect for current xDSL systems. It can be observed that the crosstalk coupling is relatively high (where a higher negative $\mathrm{dB}$ value means lower coupling and vice versa) for higher frequencies.

\subsection{MIMO Crosstalk Model}

In 2009 the Network Interface, Power, and Protection (NIPP) Committee: Network Access Interfaces (NAI) Subcommittee conducted a study [6] that provided a stochastic model for a MIMO (FEXT) coupling channel for DSL transmission systems. From this report "The model was derived using a statistical analysis of measurements of ingress energy into pairs of a cable, from other pairs in the same cable, using an actual loop plant. The probability density function (PDF) of the model is asymmetric, meaning that the crosstalk coupling from line (or pair) 1 to 2 is different from the crosstalk coupling from line 2 to 1. The difference seems to vary between $0 \mathrm{~dB}$ to $3 \mathrm{~dB}$. The MIMO crosstalk model is also based on a $99 \%$ worst case model for single or multiple binders of up to 25 pairs each (up to 100 pairs total), $0.4 \mathrm{~mm}$ or $0.5 \mathrm{~mm}$ cable type. The transfer function for the MIMO FEXT model is given as:

$$
H_{F E X T}(f)=\left|H_{F E X T, 99}(f)\right| . \mathrm{e}^{j \varphi(f)} \cdot 10^{\frac{X_{d B}}{20}}
$$

where $f$ is the frequency [Hz], $d$ is the coupling length [m], $H_{F E X T, 99}$ is the $99 \%$ worst-case crosstalk coupling model in linear scale, $\varphi(f)$ is the phase of the crosstalk channel transfer function and $X_{d B}$ is the amplitude offset of the crosstalk transfer function $[\mathrm{dB}]$, relative to the amplitude of the $99 \%$ worst case model. The phase $\varphi(f)$ is equal to the phase of the direct channel transfer function plus an offset:

$$
\varphi(f)=\tan ^{-1}\left(\frac{\operatorname{imag}\left(H_{\text {ins }}(f)\right)}{\operatorname{real}\left(H_{\text {ins }}(f)\right)}\right)+\varphi_{0}
$$

where the phase offset $\varphi_{0}$ is a uniformly distributed random variable over the range $[0,2 \pi]$." An interesting observation from the practical measurements was "...it is also clear that the phase of the crosstalk channel has the same slope as the phase of the direct channel". Thus, the crosstalk channels are only affected by the amplitude $X_{d B}(f)$. Also note that "...the amplitude offset $X_{d B}(f)$ can be considered to be independent of the frequency". Thus, the frequency variation of the amplitude offset is ignored and approximated by $X_{d B}$.

For this study a standard $100 \times 100$ matrix of $X_{d B}$ (representing a 100-pair cable with four 25-pair binders) will be used [6]. Row 70 (Pair $i=70$ as desired channel) had the worst profile and was used for the MIMO channel profiling. $X_{i, j}$ vs. Pair $j$ (only for the worse 64 cases) are shown in Table 1. Although the MIMO model was derived for FEXT, it is also applicable to NEXT, as $X_{i, j}$ is the crosstalk coupling between pairs $i$ and $j . H_{N E X T}(f)$ and $H_{F E X T}(f, d)$ is thus defined as:

$$
\begin{array}{r}
H_{N E X T}(f)=H_{N E X T, 99}(f) \cdot 10^{\frac{X_{d B}}{20}} \\
H_{F E X T}(f, d)=H_{F E X T, 99}(f, d) \cdot 10^{\frac{X_{d B}}{20}}
\end{array}
$$

\section{PERFORMANCE EVALUATION RESULTS}

Bit error rate (BER) analysis was first performed on the GDSL modem for QPSK, 16-QAM and 64-QAM modulation, with frequency spreading $L_{f s p}=64$, as shown in Figure 8. It should be observed that spreading does not affect the BER performance of the system. The

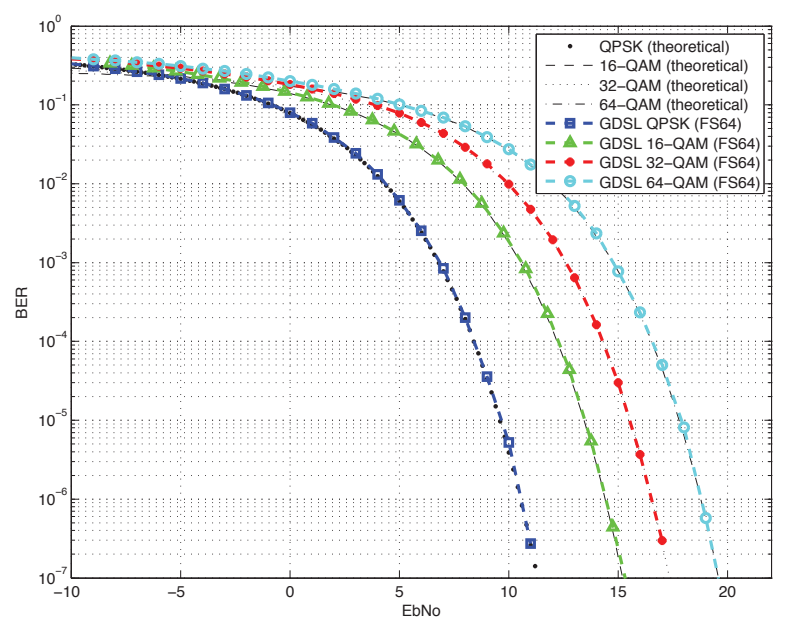

Figure 8: BER for GDSL system with $L_{f s p}=64$ frequency spreading.

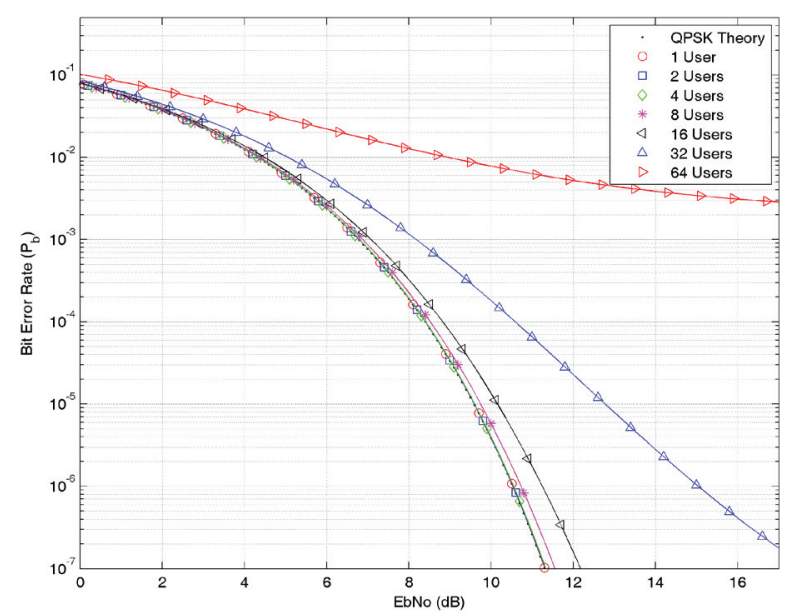

Figure 9: BER for a QPSK system for different NEXT coupled users, operating in the lowest frequency Resource Block

GDSL system was tested for different users using only 99\% worse case NEXT coupling, with operation in the lowest frequency resource block (RB), as shown in Figure 9, and in the highest frequency RB, as shown in Figure 10 ('4 Users' mean that there is the desired user and 3 


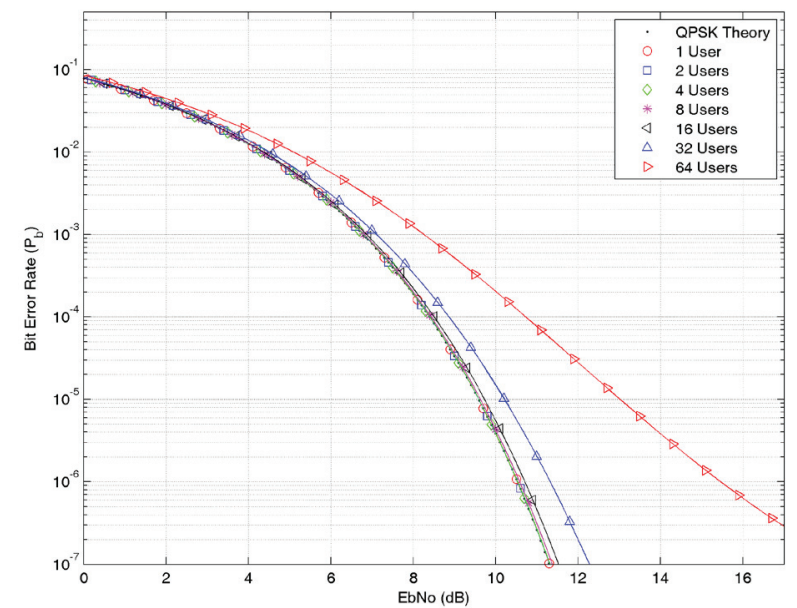

Figure 10: BER for a QPSK system for different NEXT coupled users, operating in the highest frequency Resource Block

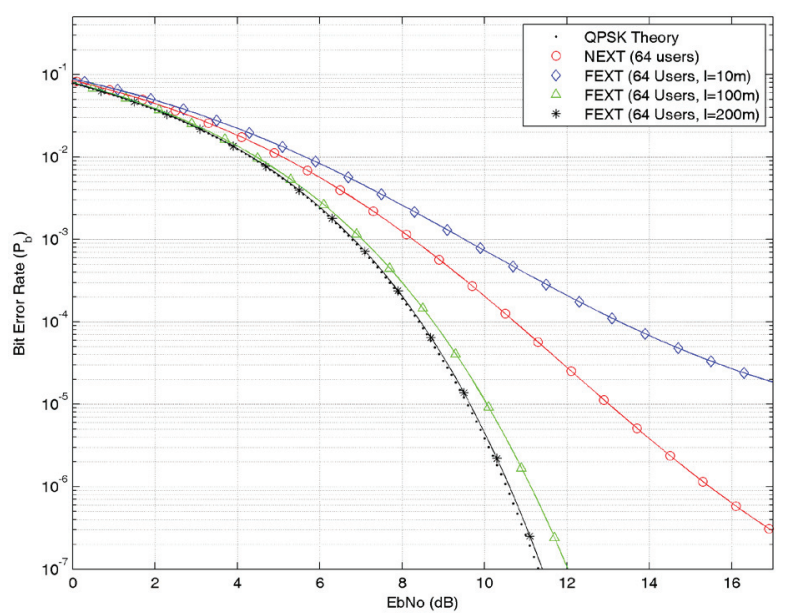

Figure 11: BER for a 64 users QPSK system, comparing NEXT and FEXT $(1=10 \mathrm{~m}, 100 \mathrm{~m}$ and $200 \mathrm{~m}$ respectively) for the high frequency RB.

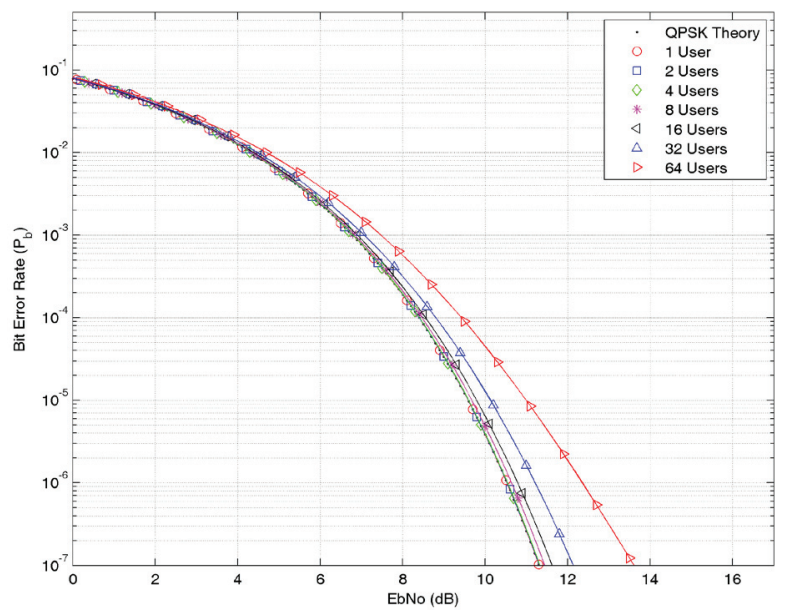

Figure 12: BER for a QPSK system for different NEXT coupled users, with practical MIMO model, operating in the lowest frequency Resource Block

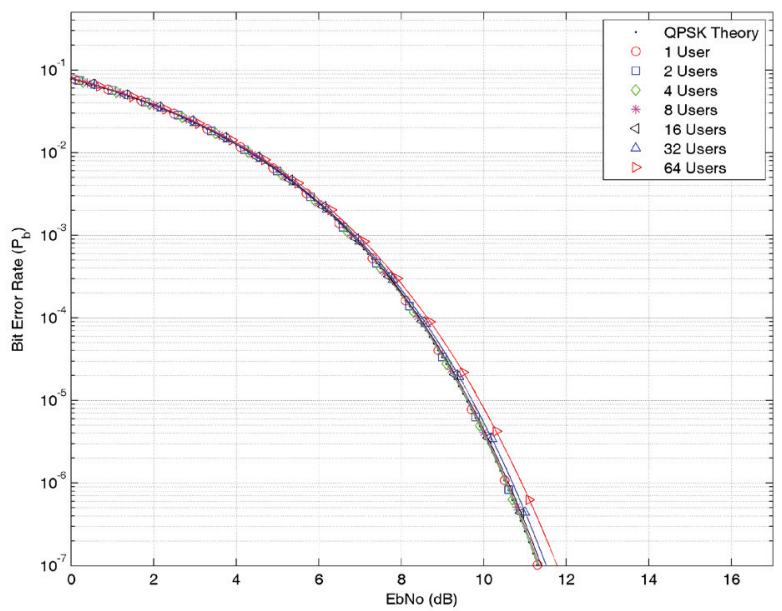

Figure 13: BER for a QPSK system for different NEXT coupled users, with practical MIMO model, operating in the highest frequency Resource Block

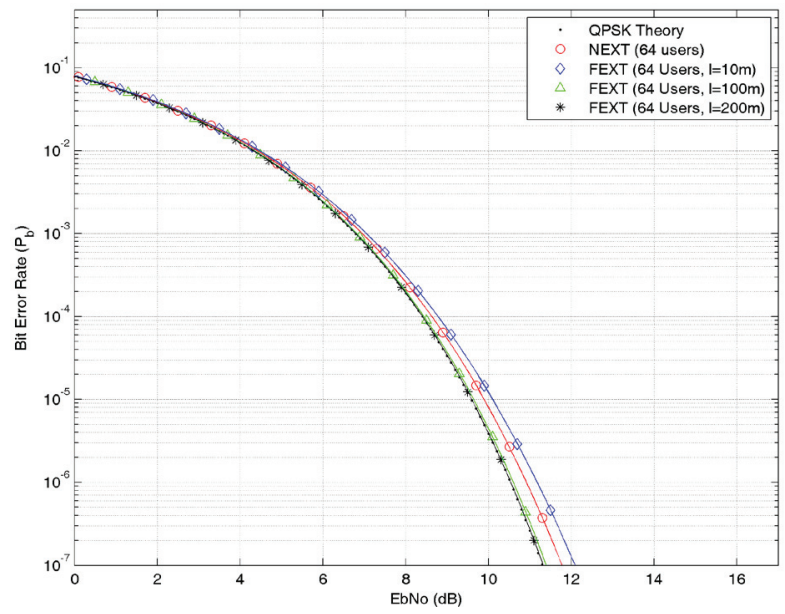

Figure 14: BER for a 64 users QPSK system, with practical MIMO model, comparing NEXT and FEXT $(1=10 \mathrm{~m}, 100 \mathrm{~m}$ and $200 \mathrm{~m}$ respectively) for the high frequency RB.

other NEXT coupled users). It can be observed that the performance for up to 16 users are still acceptable, but for up to 64 users the performance deteriorates. In Figure 11 a 64 users QPSK system is used to compare NEXT and FEXT $(1=10 \mathrm{~m}, 100 \mathrm{~m}$ or $200 \mathrm{~m}$ respectively). The system performance is acceptable for line lengths of more than $100 \mathrm{~m}$, provided that NEXT is not present.

In Section 3.3 a more practical MIMO Crosstalk model is explained. If crosstalk coupling attenuation values $\mathrm{XdB}$ of Table 1 are also taken into consideration, the performance for low - and high frequency RBs are shown in Figures 12 and 13. It can be observed that the performance for up to 64 users are still acceptable. In Figure 14 a 64 users QPSK system is used to compare NEXT and FEXT (l=10m, 100m or $200 \mathrm{~m}$ respectively) when practical crosstalk coupling attenuation $(\mathrm{XdB})$ values are taken into account. The system performance is acceptable, even for FEXT at very 
Table 1: Crosstalk coupling $X_{i, j}(\mathrm{~dB})$ for $i=70$ (worst-case)

\begin{tabular}{|c|c|c|c|c|c|c|c|c|c|c|}
\hline \multirow[t]{2}{*}{ Pair j => } & 54 & 70 & 68 & 55 & 69 & 72 & 73 & 63 & 74 & 61 \\
\hline & 0.26 & 0 & -1.07 & -2.26 & -5.56 & -7.36 & -7.79 & -7.87 & -8.4 & -8.98 \\
\hline \multirow{3}{*}{ Pair $\mathrm{j}=>$} & & & & & & & & & & \\
\hline & 77 & 39 & 65 & 96 & 27 & 99 & 34 & 81 & 66 & 87 \\
\hline & -10.41 & -11.01 & -12.06 & -12.79 & -12.96 & -13.43 & -13.51 & -13.51 & -13.54 & -14.36 \\
\hline \multirow{2}{*}{ Pair j => } & 84 & 75 & 71 & 59 & 35 & 85 & 45 & 36 & 79 & 26 \\
\hline & -14.88 & -14.98 & -15.09 & -15.34 & -15.99 & -16.63 & -17.09 & -17.3 & -17.42 & -17.71 \\
\hline \multirow{2}{*}{ Pair j => } & 20 & 8 & 19 & 91 & 32 & 82 & 6 & 44 & 76 & 98 \\
\hline & -17.92 & -18.22 & -18.22 & -18.27 & -18.83 & -19.1 & -19.56 & -19.98 & -19.99 & -20.3 \\
\hline \multirow[t]{2}{*}{ Pair j => } & 9 & 80 & 48 & 1 & 62 & 23 & 43 & 46 & 24 & 95 \\
\hline & -20.49 & -20.61 & -20.75 & -21.12 & -21.19 & -21.34 & -21.59 & -22.21 & -22.7 & -22.73 \\
\hline \multirow[t]{2}{*}{ Pair $\mathrm{j}=>$} & 52 & 58 & 5 & 78 & 3 & 33 & 64 & 28 & 13 & 97 \\
\hline & -23.03 & -23.19 & -23.37 & -23.44 & -24.27 & -24.92 & -24.96 & -24.98 & -25.03 & -25.4 \\
\hline \multirow[t]{2}{*}{ Pair j => } & 10 & 90 & 31 & 57 & & & & & & \\
\hline & -25.43 & -26.25 & -26.4 & -26.56 & & & & & & \\
\hline
\end{tabular}

short lengths or even NEXT. A unique observation from these performance tests is than the non-linear behaviour of NEXT and FEXT creates an orthogonality distortion effect on the correlation properties of the SOCC code family. The attenuation level of the relevant NEXT/FEXT function also plays a role in further amplifying this effect. This is evident when comparing Figure 9 and Figure 10, using Figure 6 to determine the degree of non-linearity (the gradient of the respective crosstalk functions at low and high frequencies). Orthogonal codes thus do not function well under high gradient changes over the spreading length of codes.

\section{CONCLUSION}

In this paper the unique performance evaluation of a GDSL modem using SOCC spreading, operating within a practical NEXT/FEXT (MIMO) channel, was described. It was found that the original $99 \%$ worst case NEXT and FEXT models are too conservative towards practical systems, as indicated in Section 3.3. Using the practically measured MIMO model, the GDSL system shows acceptable BER performance for a fully loaded system (64 users) under all NEXT/FEXT conditions.

\section{REFERENCES}

[1] van Wyk JH, Linde LP. Design of a Gigabit DSL modem using Super Orthogonal Complete Complementary Codes. Trans. Emerging Tel. Tech. (2016). Published online in Wiley Online Library (Available http://wileyonlinelibrary.com). DOI: 10.1002/ett.3071.

[2] Starr T, Cioffi JM, Silverman PJ. Understanding Digital Subscriber Line Technology. Prentice Hall. 1999. ISBN 0-13-780545-4.

[3] ITU-T. 2014. Fast access to subscriber terminals (G.fast) - Physical layer specification. Available: https://www.itu.int/rec/T-REC-G.9701/en.

[4] Leung C, Huberman S, Ho-Van K, Le-Ngoc T. Vectored DSL: Potential, Implementation Issues and Challenges. IEEE Communications \& Tutorials Q4 2013. 15 (4). pp. 1907-1923.

[5] Galli S, Kerpez K. Methods of summing crosstalk from mized sources - Part I: Theoretical Analysis. IEEE Trans. Commun., 50 (3), pp. 453461.

[6] ATIS, Multiple-input Multiple-output Crosstalk Channel Model. NIPP-NAI-2009-014R3, Tech. Rep., 2009. 